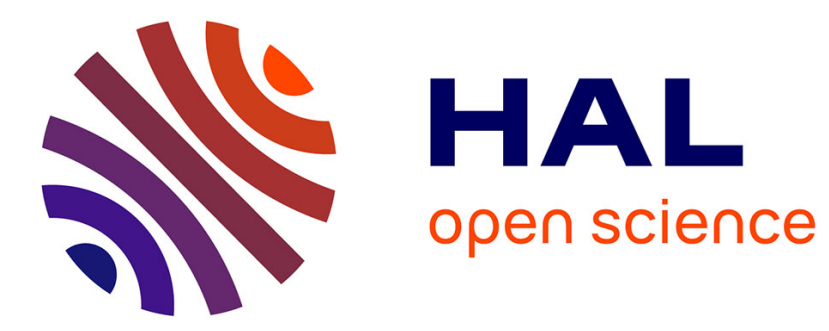

\title{
Perfect absorption of flexural waves induced by bound state in the continuum
}

\author{
Liyun Cao, Yifan Zhu, Sheng Wan, Yi Zeng, Yong Li, B. Assouar
}

\section{To cite this version:}

Liyun Cao, Yifan Zhu, Sheng Wan, Yi Zeng, Yong Li, et al.. Perfect absorption of flexural waves induced by bound state in the continuum. Extreme Mechanics Letters, 2021, 47, pp.101364. 10.1016/j.eml.2021.101364 . hal-03412439

\section{HAL Id: hal-03412439 \\ https://hal.science/hal-03412439}

Submitted on 3 Nov 2021

HAL is a multi-disciplinary open access archive for the deposit and dissemination of scientific research documents, whether they are published or not. The documents may come from teaching and research institutions in France or abroad, or from public or private research centers.
L'archive ouverte pluridisciplinaire HAL, est destinée au dépôt et à la diffusion de documents scientifiques de niveau recherche, publiés ou non, émanant des établissements d'enseignement et de recherche français ou étrangers, des laboratoires publics ou privés. 


\title{
Perfect Absorption of Flexural Waves Induced by Bound State in the Continuum
}

\author{
Liyun Cao ${ }^{1, *}$, Yifan Zhu ${ }^{1}$, Sheng $\mathrm{Wan}^{1}$, Yi Zeng ${ }^{1}$, Yong $\mathrm{Li}^{2}$ and Badreddine Assouar ${ }^{1, *}$ \\ ${ }^{1}$ Université de Lorraine, CNRS, Institut Jean Lamour, Nancy, France \\ ${ }^{2}$ Institute of Acoustics, School of Physics Science and Engineering, Tongji University, Shanghai \\ 200092, China. \\ *Corresponding author e-mails: liyun.cao@univ-lorraine.fr \& badreddine.assouar@univ-lorraine.fr
}

\begin{abstract}
Conventional vibration absorber is challenging to use in the extreme environment of high (low) temperature, due to the low tolerance of its additional damping material to temperature. To adapt to the extreme environment, here we propose an elastic metaabsorber (EMA) based on the quasi-bound states of the continuum (BICs) physical approach. The proposed absorber does not have any additional damping material, depends only on the extreme-low structural loss, and is capable of achieving perfect absorption of the flexural waves propagating in an elastic beam. The proof-of-concept we provide consists of three parallel subwavelength sub-beams sharing a single-port radiating channel in a host beam, and supports elastic Friedrich-Wintgen quasi-BICs. Based on the latter, the subwavelength EMA can achieve a high quality factor in the elastic wave system to enhance, in an unprecedented way, the wave energy dissipation. Using this physical mechanism, we experimentally and numerically validate and demonstrate the perfect absorption of flexural waves at subwavelength regime. Our work opens a new route to deal with vibration absorption with subwavelength structures in specific and extreme environments.
\end{abstract}

Keyword: Bound state in the continuum (BIC); Flexural wave; Perfect absorption 


\section{Introduction}

Mechanical vibration absorption of beam-like or plate-like structures is of considerable significance in many engineering applications, such as preventing vibration hazards and reducing noise. Conventional vibration absorption methods [13], which are represented by attaching a large number of damping on the beam-like or plate-like structures, do not fulfill the requirements of lightweight and low-frequency features. As a newly generated method of vibration absorption, the acoustic black hole $(\mathrm{ABH})$ [4-8] can absorb propagating waves (dominated by flexural waves) by little attached damping material, which promotes the development of vibration absorption technique with a lightweight structure. However, the $\mathrm{ABH}$ is not useful for technological applications at low frequencies due to the weak damping effect. Recently, vibration absorption techniques based on the new method of designing open lossy resonators in one-dimensional elastic beams have been proposed [9]. Afterwards, we have proposed the idea of lossy gradient elastic metasurface [10] in two-dimensional elastic plates and experimentally demonstrated broadband vibration absorption in low frequency. However, almost all of the vibration absorption methods mentioned above require additional damping to enhance the loss of the designed structures. These damping materials have a low tolerance to temperature, making these absorption methods challenging to apply to extreme environments with high or low temperature.

On the other hand, bound states in the continuum (BICs), a general wave phenomenon identified in different materials and wave systems, are non-decaying localized modes embedded within the continuous spectrum of radiating waves $[11,12]$. 
These states, defying the conventional bound states located outside the continuum, are also considered as trapped modes with infinite quality factors (infinite-Q) residing inside the continuum $[11,12]$. Structures with BICs supporting natural high-Q have opened the route to many practical applications in optics and photonics, such as lasers [13-16], sensors [17-19], and filters [20-22]. For the subsequent acoustic wave systems, parallel plates in a wind tunnel supporting symmetry-protected BICs [23-25] and waveguides with side-branched cavities supporting the Fabry-Pérot BICs [26, 27] have been investigated. Recently, sound confinement with an arbitrarily high quality factor was realized by designing two adjacent resonators supporting a Friedrich-Wintgen quasi-BIC [28]. So far, BICs in elastic wave systems [29] are rarely recognized and concerned due to the high sophisticated polarization states $[30,31]$ varying with the complex media structures.

In the present research, by realizing elastic Friedrich-Wintgen quasi-BIC, we introduce the concept of elastic meta-absorber (EMA) capable of perfectly absorbing the flexural waves in an extreme-low-loss elastic beam. First, we establish the theoretical model by using the temporal coupled-mode method in the elastic wave system to obtain the elastic quasi-BIC. Second, based on the quasi-BIC, we theoretically design the EMA. In addition, the physical mechanism of the perfect absorption of the EMA is uncovered and discussed. Finally, the experimental and simulation results are presented to validate the perfect absorption functionality.

\section{Theoretical model and design of EMA}

Figure 1(a) shows the proposed EMA which consists of three parallel sub-beams 
on the right edge of the host beam. They are classified into two types, i.e., sub-beam A and sub-beam $\mathrm{B}$, which are the outer ones with the length $d_{\mathrm{A}}$ and the middle one with the length $d_{\mathrm{B}}$, respectively, as shown in Fig. 1(a). The whole structure is symmetrical to the central axis [the purple dotted line in Fig. 1(a)]. Note that if the two outer sub-beams are different, i.e., asymmetric to the central axis, the torsion mode in the host beam will be induced by the moment from the different shear forces between the two outer subbeams. In our system with symmetrical structure, there is no fundamental difference between three or more sub-beams sharing a single-port radiating channel in the host beam with non-reflection boundary on the left edge. The material parameters of all models are consistent with that of the experimental 3D-printed material PLA. The density and Poisson's ratio are $\rho=1086.3 \mathrm{~kg} / \mathrm{m}^{3}[29]$ and $v=0.35$, respectively. Young's modulus is $E=3.44 \times 10^{9} \times(1+0.015 \times \mathrm{i}) \mathrm{Pa}$, and the small imaginary part, which is obtained by fitting the simulated resonance peaks with the experimental ones, corresponds to the low structural loss. Only flexural waves in these elastic beams are considered in the whole study, because there is no mode conversion.

(a)

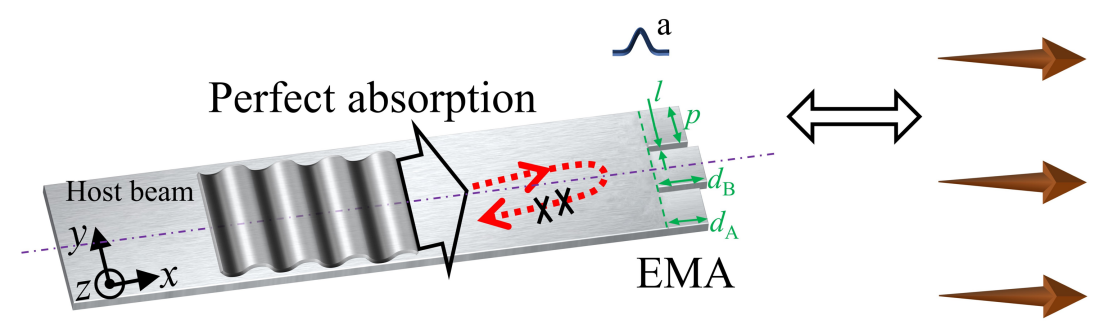

(b)

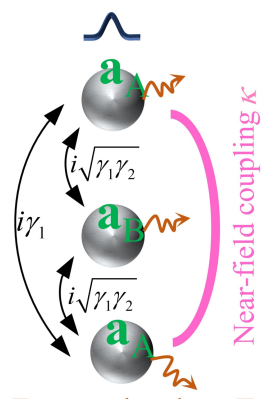

Extreme-low loss $\Gamma$

Fig. 1(a) The elastic meta-absorber (EMA) consists of three parallel sub-beams on the right edge of the host beam with the thickness of $h=1 \mathrm{~mm}$. These sub-beams have the same width of $p=3 \mathrm{~mm}$, 
which is less than a quarter wavelength in the target frequency range. The separation between adjacent sub-beams of $l$ is $0.5 \mathrm{~mm}$. (b) Schematic illustration for the set-up of temporal coupledmode theory. The sub-beam resonators A and B have the amplitude $\mathbf{a}_{\mathrm{A}}$ and $\mathbf{a}_{\mathrm{B}}$, respectively.

First, the EMA model in Fig. 1(a) degenerates to the simplified ones with the same sub-beam length, i.e., including only sub-beam A or B. They are marked as the degenerated model A (DMA) and the degenerated model B (DMB), respectively. We obtain the absorption curves of these degenerated models by experiments [orange balls and blue-green balls in Fig.3(b)] and frequency-domain simulations [orange line and blue-green line in Fig.3(b)] based on solid mechanics module of COMSOL Multiphysics 5.4 software [10]. The corresponding resonance frequencies $\omega_{\mathrm{A}}$ and $\omega_{\mathrm{B}}$ are extracted from the peaks of the absorption curves. These resonances are from the coupling between the scattering fields of the sub-beams and the host beam boundaries. In classical mechanics, elastic beams are often simplified as onedimensional models, but our system is not suitable for this simplification due to the coupling. Note that, when the host beam equivalent to a plate model by applying periodic boundary conditions, the scattering fields from the host beam boundaries disappear, thus these resonances will disappear. For the EMA model in Fig. 1(a), each sub-beam resonator (A and $\mathrm{B}$ ) can be described by the temporal coupled-mode equation [schematic illustration in Fig. 1(b)] for the lowest mode with amplitude $\mathbf{a}_{\mathrm{A}(\mathrm{B})}=a_{\mathrm{A}(\mathrm{B})} \cdot e^{\mathrm{i} \omega t}$ as [11]

$$
\frac{\mathrm{d}}{\mathrm{d} t} \mathbf{a}_{\mathrm{A}}=\left(\mathrm{i} \omega_{\mathrm{A}}-\gamma_{\mathrm{A}}-\Gamma\right) \mathbf{a}_{\mathrm{A}}+\mathrm{i} \kappa \mathbf{a}_{\mathrm{B}}+\mathrm{i} \sqrt{\gamma_{\mathrm{A}}}\left(S_{i}+\mathrm{i} \sqrt{\gamma_{\mathrm{A}}} \mathbf{a}_{\mathrm{A}}+\mathrm{i} \sqrt{\gamma_{\mathrm{B}}} \mathbf{a}_{\mathrm{B}}\right)
$$




$$
\frac{\mathrm{d}}{\mathrm{d} t} \mathbf{a}_{\mathrm{B}}=\left(\mathrm{i} \omega_{\mathrm{B}}-\gamma_{\mathrm{B}}-\Gamma\right) \mathbf{a}_{\mathrm{B}}+\mathrm{i} \kappa \mathbf{a}_{\mathrm{A}}+\mathrm{i} \sqrt{\gamma_{\mathrm{B}}}\left(S_{i}+2 \mathrm{i} \sqrt{\gamma_{\mathrm{A}}} \mathbf{a}_{\mathrm{A}}\right)
$$

where $S_{i}$ represents the incident wave, $\gamma_{\mathrm{A}(\mathrm{B})}$ is the corresponding radiative decay rate, and $\Gamma$ represents the dissipative decay rate associated with the low structural loss. The terms $i \kappa \mathbf{a}_{\mathrm{A}}$ and $i \kappa \mathbf{a}_{\mathrm{B}}$ describe the near-field coupling between two types of resonators emanating from their evanescent flexural wave modes. The terms $i \sqrt{\gamma_{\mathrm{A}}} \mathbf{a}_{\mathrm{A}}$ and $i \sqrt{\gamma_{\mathrm{B}}} \mathbf{a}_{\mathrm{B}}$ in Eq. (1) represent the reradiation field of resonator A caused by the other two resonators. The term $2 i \sqrt{\gamma_{\mathrm{A}}} \mathbf{a}_{\mathrm{A}}$ in Eq. (2) represents the reradiation field of resonator B caused by the other two resonators.

In the absence of incident wave and intrinsic loss, Eqs. (1) and (2) can be derived as $-\mathrm{i} \partial A / \partial t=\mathbf{H} A$, where $A=\left[\mathbf{a}_{\mathrm{A}}, \mathbf{a}_{\mathrm{B}}\right]^{\mathrm{T}}$ includes two types of mode amplitudes. The Hamiltonian $\mathbf{H}$ can be expressed as

$$
\mathbf{H}=\left(\begin{array}{cc}
\omega_{\mathrm{A}} & 0 \\
0 & \omega_{\mathrm{B}}
\end{array}\right)+\mathrm{i}\left(\begin{array}{cc}
2 \gamma_{\mathrm{A}} & \sqrt{\gamma_{\mathrm{A}} \gamma_{\mathrm{B}}}-\mathrm{i} \kappa \\
2 \sqrt{\gamma_{\mathrm{A}} \gamma_{\mathrm{B}}}-\mathrm{i} \kappa & \gamma_{\mathrm{B}}
\end{array}\right)
$$

The two eigenvalues of $\mathbf{H}$ can be obtained as

$$
\bar{\omega}=\frac{\omega_{\mathrm{A}}+2 \mathrm{i} \gamma_{\mathrm{A}}+\omega_{\mathrm{B}}+\mathrm{i} \gamma_{\mathrm{B}}}{2} \pm \frac{\sqrt{\left(\omega_{\mathrm{A}}+2 \mathrm{i} \gamma_{\mathrm{A}}-\omega_{\mathrm{B}}-\mathrm{i} \gamma_{\mathrm{B}}\right)^{2}-4\left(\sqrt{\gamma_{\mathrm{A}} \gamma_{\mathrm{B}}}-\mathrm{i} \kappa\right)\left(2 \sqrt{\gamma_{\mathrm{A}} \gamma_{\mathrm{B}}}-\mathrm{i} \kappa\right)}}{2}
$$

When the lengths of two resonators satisfy $\Delta d=d_{\mathrm{B}}-d_{\mathrm{A}}=0$, they have the same resonance frequency of $\omega_{\mathrm{A}}=\omega_{\mathrm{B}}=\omega$, the same radiative decay rate of $\gamma_{\mathrm{A}}=\gamma_{\mathrm{B}}=\gamma$, and the vanished near-field interaction $\kappa=0$. Therefore, according to Eq. (4), one eigenvalue $\bar{\omega}$ becomes $\omega+3 \gamma$ i with more loss, while the other becomes a purely real $\omega$ without any loss. The latter indicates that the system turns into the BIC [11] with an infinite Q. To intuitively display the BIC, the reflection coefficient of the system, $r$, can be expressed as 


$$
r=1+4 \mathrm{i} \sqrt{\gamma_{\mathrm{A}}} \frac{a_{\mathrm{A}}}{S_{i}}+2 \mathrm{i} \sqrt{\gamma_{\mathrm{B}}} \frac{a_{\mathrm{B}}}{S_{i}}
$$

The first term on the right-hand side of Eq. (5) describes the reflection of the incident wave reflected by the right boundary in the background field. The second and third terms describe the reflections of flexural waves from resonator $\mathrm{A}$ and resonator $\mathrm{B}$, respectively. Factors 4 and 2 occur in Eq. (5) because one resonator A(B) has the same left-radiation and right-radiation, i.e., $i \sqrt{\gamma_{A}} a_{\mathrm{A}}\left(\mathrm{i} \sqrt{\gamma_{\mathrm{B}}} a_{\mathrm{B}}\right)$. Due to the reflection of rightradiation in the right boundary, as shown in Fig. 1(a), the resonator A(B) will have the left-radiation of $2 \mathrm{i} \sqrt{\gamma_{A}} a_{\mathrm{A}}\left(2 \mathrm{i} \sqrt{\gamma_{\mathrm{B}}} a_{\mathrm{B}}\right)$. Based on Eqs. (1), (2), and (5), the dimensionless reflected energy coefficient $|r|^{2}$ of the system varying with the length of resonator $\mathrm{B} d_{\mathrm{B}}=d_{\mathrm{A}}+\Delta d \quad\left(d_{\mathrm{A}}\right.$ is fixed to $7 \mathrm{~mm}$ ) is shown in Fig. 2(a). Near $\Delta d / h=0.15$ and $4.14 \mathrm{kHz}$, it shows a vanishing linewidth (VL), which is a typical characterization of the ideal BIC with infinite-Q [11, 12]. The VL position corresponding to $\Delta d / h=0.15$ has a small discrepancy with its theory position corresponding to $\Delta d / h=0$ (i.e, $d_{\mathrm{A}}=d_{\mathrm{B}}$ ). This discrepancy is expected, because the intrinsic structural loss is considered in calculating $|r|^{2}$ to show VL. 
(a)

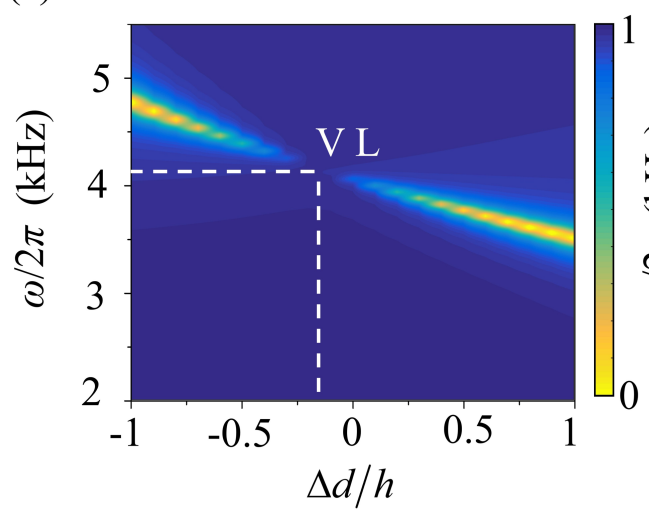

(c)

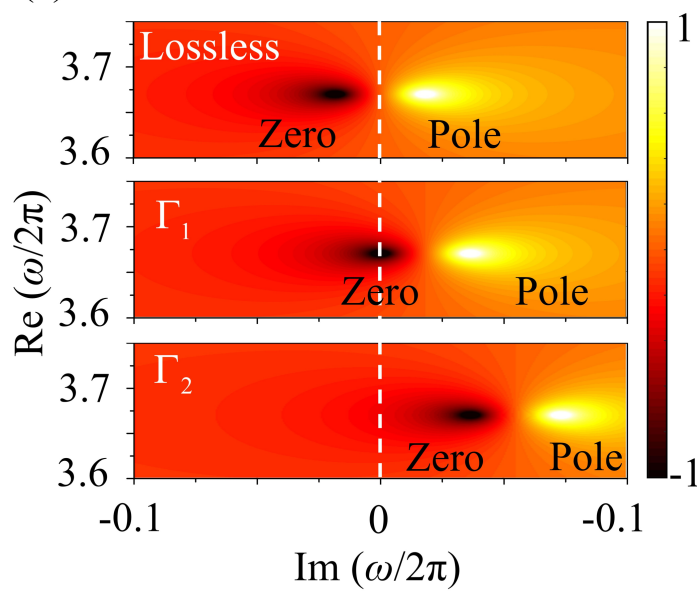

(b)

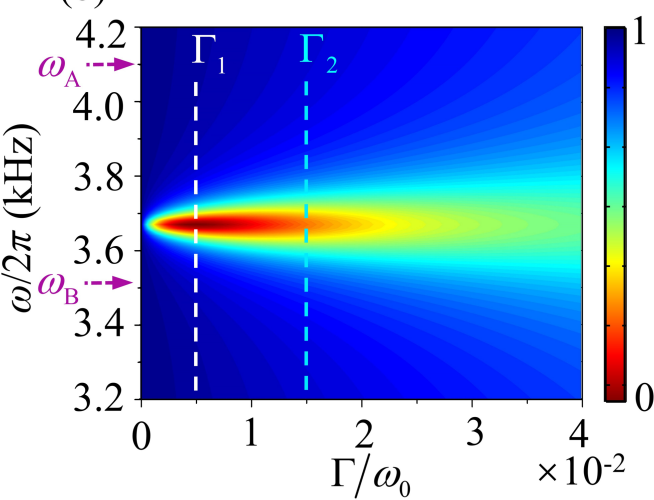

(d)

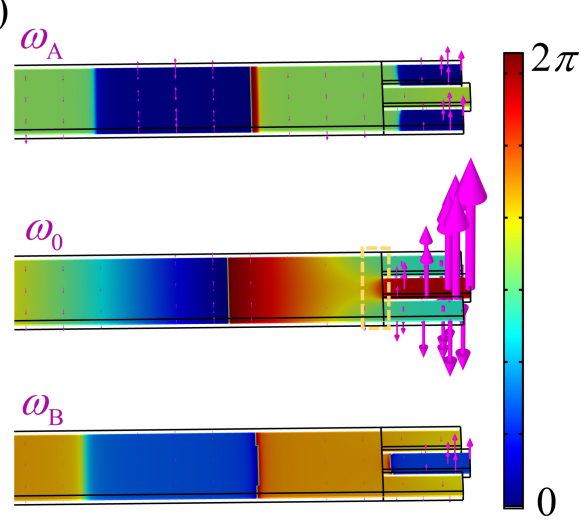

Fig. 2 (a) Dimensionless reflected energy coefficient $|r|^{2}$ of the system varying with the length difference of the sub-beams $\Delta d=d_{\mathrm{B}}-d_{\mathrm{A}}$ and the frequency $\omega / 2 \pi . \quad \gamma_{\mathrm{A}}=0.342 \omega_{\mathrm{A}}, \gamma_{\mathrm{B}}=0.302 \omega_{\mathrm{B}}$, $\Gamma / \omega_{0}=0.005$, and $\kappa=24$ are extracted from eigen-frequency simulations [28] to fit the simulated results. (b) The absorption coefficient $\alpha=1-|r|^{2}$ varying with the structural loss $\Gamma / \omega_{0}$ and the frequency $\omega / 2 \pi$. Purple arrows refer to the resonant frequencies of individual sub-beam A $\left(\omega_{\mathrm{A}} / 2 \pi=4.1 \mathrm{kHz}\right)$ and sub-beam B $\left(\omega_{\mathrm{B}} / 2 \pi=3.5093 \mathrm{kHz}\right)$. (c) The distributions of $\log _{10}|r|$ in the complex frequency planes are shown for the systems with lossless, the structural loss $\Gamma_{1}$, and the structural loss $\Gamma_{2}$, respectively. (d) The phase distributions of flexural waves in the neutral planes of the structures for three frequencies, i.e., $\omega_{\mathrm{A}}, \omega_{\mathrm{B}}$, and $\omega_{0}$. The direction and size of the pink arrows indicate the displacement direction and amplitude of the particle displacements, respectively. 
The ideal BIC $(\Delta d / h=0.15)$ with infinite-Q is completely bound and cannot be directly coupled with the external radiation channel $[11,12]$. To achieve perfect absorption of incident flexural waves from the external channel, we introduce a slight deviation to the length of resonator $\mathrm{B}(\Delta d / h$ is tun to 0.7$)$. The deviation will make the resonance system radiates a small amount of energy and couples it to the external channel. This process turns the ideal BIC into the quasi-BIC $[28,32,33]$ with a high Q. In this case, the real eigenvalue of the ideal BIC turns to complex with a tiny imaginary part indicated as the radiative decay rate of the system, $\gamma_{S}$. The perfect absorption mode, manifested by the absorption coefficient of $\alpha=1-|r|^{2} \approx 1$, appears at the frequency $\left(\omega_{0} / 2 \pi=3.67 \mathrm{kHz}\right)$ between $\omega_{\mathrm{A}}$ and $\omega_{\mathrm{B}}$ with a critical low structure $\operatorname{loss}\left(\Gamma_{1} / \omega_{0}=5 \times 10^{-3}\right)$, as shown in Fig. $2(\mathrm{~b})$.

In addition, in a complex frequency plane, the distribution of $\log _{10}|r|$ can be plotted for the system with lossless, the structural loss $\Gamma_{1}$, and the structural loss $\Gamma_{2}$, respectively, as shown in Fig. 2(c). For the system with the low structure loss $\Gamma_{1}$, "Zero" point intersects the real frequency axis. It indicates that the dissipative decay rate of the system $\left(\Gamma_{1}\right)$ is tuned to the same value as $\gamma_{S}$, which is equivalent to the so-called critical coupling $[34,35]$, however here it is fulfilled by the small $\Gamma_{1}$ and $\gamma_{S}$. The incident flexural waves can be completely absorbed without any backscattering. Note that, theoretically, we can obtain a perfect absorption for the specific frequency in the extreme environment with any high (low) temperature by adjusting the structural parameters to make $\gamma_{S}$ equal to a different $\Gamma$. Furthermore, we extract the phase distribution of the neutral plane of the structure for the three resonance frequencies, i.e., 
$\omega_{\mathrm{A}}, \omega_{\mathrm{B}}$, and $\omega_{0}$, respectively, as shown in Fig. 2(d). For the resonance frequency of $\omega_{0}$, we can see from the dotted box in Fig. 2(d) that the phase difference between the adjacent sub-beams is $\pi$, which manifests the strong coupling behavior between these sub-beams [36]. The directions and sizes of the pink arrows in Fig. 2(d) indicate the directions and amplitudes of the particle displacements, respectively. It can be observed for the resonance frequency of $\omega_{0}$ that the displacement amplitudes inside the whole sub-beams are greatly enhanced, which is caused by the strong coupling. The large enhanced displacements in the sub-beams lead to the perfect absorption of the EMA, although the structural loss is low.

\section{Experimental demonstration of perfect absorption of flexural waves}

To confirm our theory, we have printed the specimens by 3D-printer, as shown in Fig. 3(a). A 15-mm-diameter circular PZT patch is bonded on the beam surface. The PZT patch is driven by a signal generator (Tektronix AFG3022C). The blue-tack layer is bonded in the left boundary of the specimen, which is equivalent to a non-reflection boundary. Point 1 and point 2 marked in Fig. 3(a) are two measuring points. The distance between the two points is less than an eighth wavelength. Simultaneously, their locations and the boundary of the resonance structure meet the far-field assumption [10]. The assumption ensures that the measurement wavefield of the two points can approximate the sum of incident and reflection propagating waves.

Using the measurement mode "FFT" of Polytec Scanning Vibrometer 500 (PSV500), the complex velocities of flexural waves in points 1 and 2 can be measured. These measured velocities are expressed as $v^{1}=V_{\mathrm{I}} \cdot e^{\mathrm{i}\left(\bar{s}_{1}+\bar{s}_{2}\right) \cdot \hat{k}_{b}}+V_{\mathrm{R}} \cdot e^{-\mathrm{i}\left(\bar{s}_{1}+\bar{s}_{2}\right) \cdot \hat{k}_{b}} \quad$ and 
$v^{2}=V_{\mathrm{I}} e^{\mathrm{i} s_{2} \cdot \hat{k}_{b}}+V_{\mathrm{R}} e^{-\mathrm{i} \bar{s}_{2} \cdot \hat{k}_{b}}$, respectively. $\bar{s}_{1}$ and $\bar{s}_{2}$ are the distance, as shown in Fig. 3(a). $\hat{k}_{b}$ is the measured wavenumber of the flexural wave. The transfer function $H_{12}$ of the total wavefield in the two points can be obtained from the measured complex velocities of $v^{1}$ and $v^{2}$. The transfer function can be expressed as $H_{12}=v^{2} / v^{1}=\left(e^{\mathrm{i} \hat{k}_{b} \bar{s}_{2}}+\tilde{r} e^{-\mathrm{i} \hat{k}_{b} \bar{s}_{2}}\right) /\left[e^{\mathrm{i} \hat{k}_{b}\left(\bar{s}_{1}+\bar{s}_{2}\right)}+\tilde{r} e^{-\mathrm{i} \hat{k}_{b}\left(\bar{s}_{1}+\bar{s}_{2}\right)}\right]$, where the reflection coefficient is $\tilde{r}=V_{\mathrm{R}} / V_{\mathrm{I}}$. Then, we can calculate the reflection coefficient of flexural waves as

$$
\tilde{r}=\left(H_{12}-e^{-\mathrm{i} \hat{k}_{b} \bar{s}_{1}}\right) \cdot e^{\mathrm{i} 2\left(\bar{s}_{1}+\bar{s}_{2}\right) \cdot \hat{k}_{b}} /\left(e^{-\mathrm{i} \hat{k}_{b} \bar{s}_{1}}-H_{12}\right)
$$

The absorption coefficient of flexural waves can be calculated by $\tilde{\alpha}=1-|\tilde{r}|^{2}$.

(a)

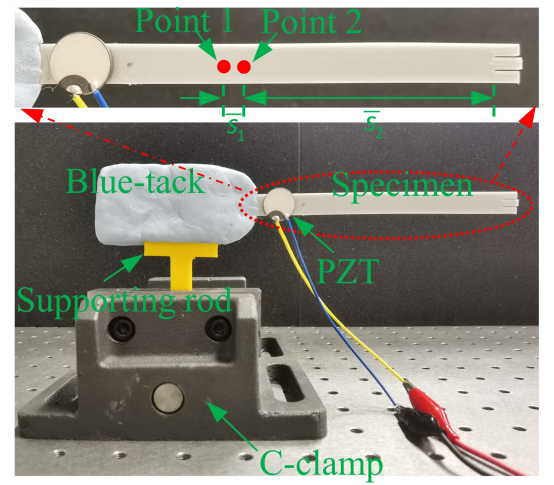

(c) (b)

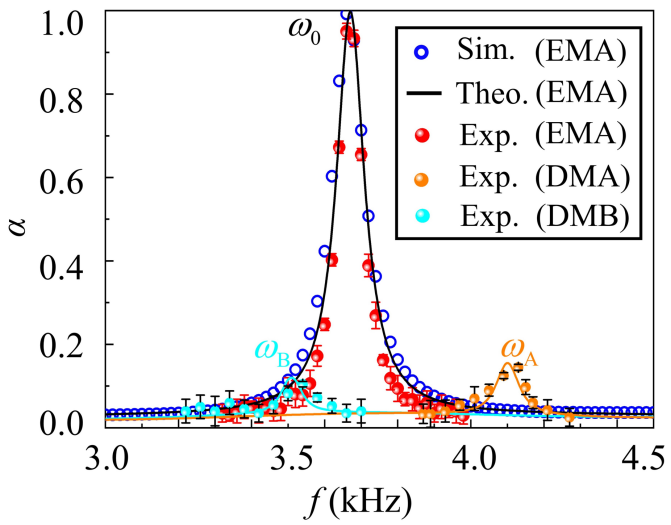

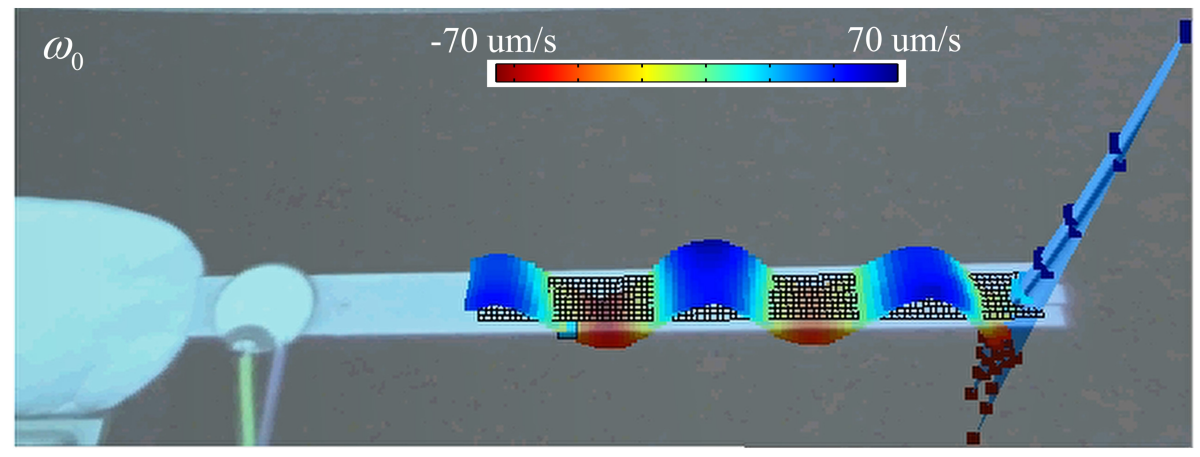

Fig. 3 (a) The specimen and test set-up. (b) For the absorption coefficient $\alpha$, the experiment results with error bars are shown by red balls. For comparison, theoretical and numerical results are displayed by the black line and blue circle, respectively. The absorption curves of DMA and DMB are also shown by the experiments (orange balls and blue-green balls) and the frequency-domain 
simulations (orange line and blue-green line), respectively. (c) The snapshots of Supplementary Video 1 from PSV 500 for the central frequency of $3.66 \mathrm{kHz}$.

To ensure the reliability of the data, we tested each experimental data three times. The experiment results with error bars are shown by red balls in Fig. 3(b). It can be seen that the peak values at $3.66 \mathrm{kHz}$ are approximately one, that is, the flexural waves are almost completely absorbed. The absorption coefficient curves from the theory [solid black lines in Fig. 3(b)] and simulations [blue circles in Fig. 3(b)] present an excellent agreement with that from the experiments. To intuitively show the absorption, we obtain the dynamic full wavefield in the specimen at the resonance frequency of 3.66 kHz by the "time"' measurement mode. Figs. 3(c) shows a snapshot of Supplementary Video 1 at $44.424 \mathrm{~ms}$. It can be clearly seen that the displacement deformations inside the whole sub-beams are greatly enhanced, which is consistent with the enhanced displacement amplitude in Fig. 2(d). In addition, in the test area, the incident wave is a perfect traveling wave, whose intuitive evidence can be found in Supplementary Video 1. The existence of the perfect traveling wave means that the EMA, indeed, perfectly absorbs flexural waves. For comparison, we also test the dynamic full wavefield at 3.2 $\mathrm{kHz}$ (away from the central frequency), as shown in Supplementary Video 2. The intuitive evidence from Supplementary Video 2 is the incident wave is a standing wave and the displacement deformations inside the sub-beams are not enhanced. 


\section{The influence of the structural parameters on the absorption}

We have also investigated the influence of the structural parameters ( $p$ and $l$ ), which are not discussed above, on the absorption spectra. Figure 4(a) shows the absorption spectrum as a function of the sub-beam width, $p$, with other parameters fixed. It is found that with the increase in $p$, the absorption effect has strong robustness, and the absorption peak shifts to low frequency. The reason is that the resonant frequency of the sub-beams becomes lower when increasing $p$, leading to a lower frequency of quasi-BIC from the coupling between sub-beams.

Figure 4(b) illustrates the absorption spectrum as a function of the separation between adjacent sub-beams, $l$, with other parameters fixed. It is found that with the increase in $l$, the absorption peak shifts to low frequency. We know that FriedrichWintgen quasi-BIC is caused by the coupling interferences of different resonance radiations at the same location [11]. In other words, for the quasi-BIC, the separation between adjacent resonators needs to be very small compared to the incident wavelength to approximate the same position. When the separation becomes large to a certain extent [less than $3.5 \mathrm{~h}$ shown in Fig. 4(b)], a larger wavelength can make adjacent sub-beams close to the same position to support the quasi-BIC, so the absorption peak based on the quasi-BIC shifts to low frequency. However, when the separation is large enough [more than $3.5 \mathrm{~h}$ shown in Fig. 4(b)], the coupling interferences between adjacent sub-beams becomes weak, and the quasi-BIC disappears, thus the absorption coefficient becomes small. 
(a)

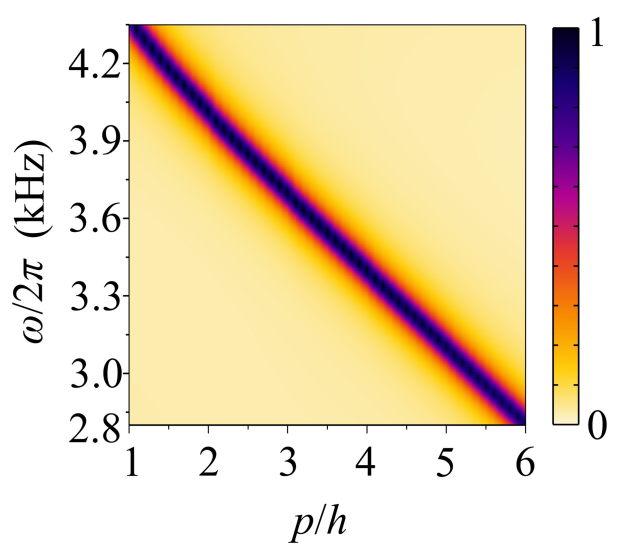

(b)

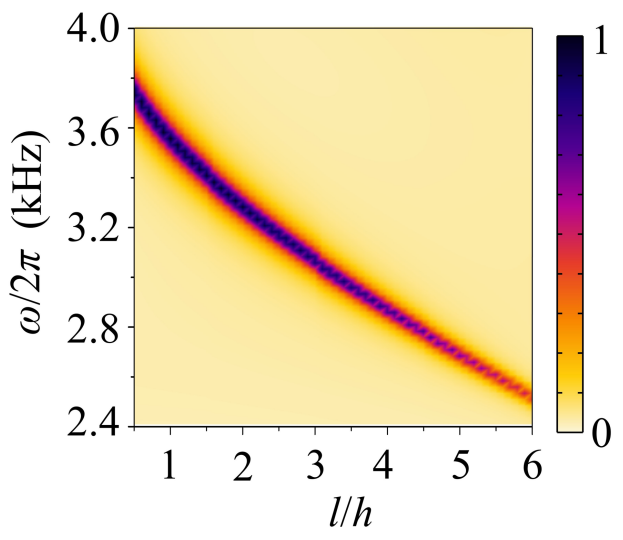

Fig. 4 (a) The absorption spectrum as a function of the sub-beam width, $p$, with other parameters fixed $\left(l=0.5 \mathrm{~mm}, d_{\mathrm{A}}=7 \mathrm{~mm}, d_{\mathrm{B}}=7.7 \mathrm{~mm}\right.$, and $\left.h=1 \mathrm{~mm}\right)$. (b) The absorption spectrum as a function of the separation between adjacent sub-beams, with other parameters fixed ( $p=3 \mathrm{~mm}, d_{\mathrm{A}}$ $=7 \mathrm{~mm}, d_{\mathrm{B}}=7.7 \mathrm{~mm}$, and $h=1 \mathrm{~mm}$ ). The bar color indicates the value of the absorption coefficient.

\section{Conclusion}

We have theoretically designed, numerically, and experimentally demonstrated the sub-wavelength EMA composed of parallel sub-beam resonators. By realizing Friedrich-Wintgen quasi-BIC in the elastic wave system, the EMA can obtain a high-Q to enhance, in an unprecedented way, the energy dissipation. The EMA perfectly absorbs the incident flexural waves in the elastic beam when the low structure loss compensates an identically low radiation decay rate. The length of the EMA is only a quarter of the target wavelength and can be further reduced by increasing the width of the sub-beams or the separation between adjacent sub-beams. Significantly, the EMA does not require any additional damping material, which could open up possibilities in the families of elastic subwavelength meta-absorber to realize low-frequency perfect absorption in the extreme environment of high (low) temperature. 


\section{Acknowledgements}

This project is supported by the Institute CARNOT (ICEEL) and la Région Grand Est.

\section{Reference}

[1] E.M. Kerwin, Damping of flexural waves by a constrained viscoelastic layer, The Journal of the Acoustical Society of America, 31 (1959) 952-962.

[2] J.Q. Sun, M.R. Jolly, M.A. Norris, Passive, adaptive and active tuned vibration absorbers-a survey, J Mech Design, 117 (1995) 234-242.

[3] G.B. Warburton, Optimum absorber parameters for various combinations of response and excitation parameters, Earthquake Eng Struc, 10 (1982) 381-401.

[4] C.A. McCormick, M.R. Shepherd, Design optimization and performance comparison of three styles of one-dimensional acoustic black hole vibration absorbers, J Sound Vib, (2019).

[5] L. Tang, L. Cheng, H. Ji, J. Qiu, Characterization of acoustic black hole effect using a onedimensional fully-coupled and wavelet-decomposed semi-analytical model, J Sound Vib, 374 (2016) 172-184.

[6] L. Ma, L. Cheng, Topological optimization of damping layout for minimized sound radiation of an acoustic black hole plate, J Sound Vib, 458 (2019) 349-364.

[7] V.V. Krylov, R.E.T.B. Winward, Experimental investigation of the acoustic black hole effect for flexural waves in tapered plates, J Sound Vib, 300 (2007) 43-49.

[8] A. Pelat, F. Gautier, S.C. Conlon, F. Semperlotti, The acoustic black hole: A review of theory and applications, J Sound Vib, (2020).

[9] J. Leng, F. Gautier, A. Pelat, R. Picó, J.P. Groby, V. Romero-García, Limits of flexural wave absorption by open lossy resonators: reflection and transmission problems, New J Phys, 21 (2019) 053003.

[10] L. Cao, Z. Yang, Y. Xu, S.-W. Fan, Y. Zhu, Z. Chen, Y. Li, B. Assouar, Flexural wave absorption by lossy gradient elastic metasurface, J Mech Phys Solids, 143 (2020) 104052.

[11] C.W. Hsu, B. Zhen, A.D. Stone, J.D. Joannopoulos, M. Soljacic, Bound states in the continuum, Nat. Rev. Mater., 1 (2016).

[12] A. Kodigala, T. Lepetit, Q. Gu, B. Bahari, Y. Fainman, B. Kanté, Lasing action from photonic bound states in continuum, Nature, 541 (2017) 196-199.

[13] S. Noda, M. Yokoyama, M. Imada, A. Chutinan, M. Mochizuki, Polarization Mode Control of Two-Dimensional Photonic Crystal Laser by Unit Cell Structure Design, Science, 293 (2001) 11231125.

[14] H. Matsubara, S. Yoshimoto, H. Saito, Y. Jianglin, Y. Tanaka, S. Noda, GaN Photonic-Crystal Surface-Emitting Laser at Blue-Violet Wavelengths, Science, 319 (2008) 445-447.

[15] K. Hirose, Y. Liang, Y. Kurosaka, A. Watanabe, T. Sugiyama, S. Noda, Watt-class high-power, high-beam-quality photonic-crystal lasers, Nat Photonics, 8 (2014) 406-411.

[16] M. Imada, S. Noda, A. Chutinan, T. Tokuda, M. Murata, G. Sasaki, Coherent two-dimensional lasing action in surface-emitting laser with triangular-lattice photonic crystal structure, Appl Phys Lett, 75 (1999) 316-318. 
[17] A.A. Yanik, A.E. Cetin, M. Huang, A. Artar, S.H. Mousavi, A. Khanikaev, J.H. Connor, G. Shvets, $\mathrm{H}$. Altug, Seeing protein monolayers with naked eye through plasmonic Fano resonances, Proceedings of the National Academy of Sciences, 108 (2011) 11784-11789.

[18] S. Romano, G. Zito, S.N. Lara Yépez, S. Cabrini, E. Penzo, G. Coppola, I. Rendina, V. Mocellaark, Tuning the exponential sensitivity of a bound-state-in-continuum optical sensor, Opt Express, 27 (2019) 18776-18786.

[19] B. Zhen, S.-L. Chua, J. Lee, A.W. Rodriguez, X. Liang, S.G. Johnson, J.D. Joannopoulos, M. Soljačić, O. Shapira, Enabling enhanced emission and low-threshold lasing of organic molecules using special Fano resonances of macroscopic photonic crystals, Proceedings of the National Academy of Sciences, 110 (2013) 13711-13716.

[20] J.M. Foley, S.M. Young, J.D. Phillips, Symmetry-protected mode coupling near normal incidence for narrow-band transmission filtering in a dielectric grating, Phys Rev B, 89 (2014) 165111.

[21] L.L. Doskolovich, E.A. Bezus, D.A. Bykov, Integrated flat-top reflection filters operating near bound states in the continuum, Photonics Research, 7 (2019) 1314.

[22] C.-Y. Ju, M.-H. Chou, G.-Y. Chen, Y.-N. Chen, Optical quantum frequency filter based on generalized eigenstates, Opt Express, 28 (2020) 17868-17880.

[23] R. Parker, W.M. Griffiths, Low frequency resonance effects in wake shedding from parallel plates, J Sound Vib, 7 (1968) 371-379.

[24] W. Koch, Resonant acoustic frequencies of flat plate cascades, J Sound Vib, 88 (1983) 233242.

[25] N.A. Cumpsty, D.S. Whitehead, The excitation of acoustic resonances by vortex shedding, J Sound Vib, 18 (1971) 353-369.

[26] A.A. Lyapina, A.S. Pilipchuk, A.F. Sadreev, Trapped modes in a non-axisymmetric cylindrical waveguide, J Sound Vib, 421 (2018) 48-60.

[27] S. Hein, W. Koch, L. Nannen, Trapped modes and Fano resonances in two-dimensional acoustical duct-cavity systems, J Fluid Mech, 692 (2012) 257-287.

[28] S. Huang, T. Liu, Z. Zhou, X. Wang, J. Zhu, Y. Li, Extreme Sound Confinement From Quasibound States in the Continuum, Phys Rev Appl, 14 (2020).

[29] L. Cao, Y. Zhu, Y. Xu, S. -W. Fan, Z. Yang, B. Assouar, Elastic Bound State in the Continuum with Perfect Mode Conversion, 2021, pp. arXiv:2103.09006.

[30] J.L. Rose, Ultrasonic waves in solid media, Cambridge University Press, 10 (1999).

[31] K.F. Graff, Wave motion in elastic solids, 1975.

[32] Z. Liu, Y. Xu, Y. Lin, J. Xiang, T. Feng, Q. Cao, J. Li, S. Lan, J. Liu, High-\$Q\$ Quasibound States in the Continuum for Nonlinear Metasurfaces, Phys Rev Lett, 123 (2019) 253901.

[33] K. Koshelev, S. Lepeshov, M. Liu, A. Bogdanov, Y. Kivshar, Asymmetric Metasurfaces with High\$Q\$ Resonances Governed by Bound States in the Continuum, Phys Rev Lett, 121 (2018) 193903. [34] M. Cai, O. Painter, K.J. Vahala, Observation of Critical Coupling in a Fiber Taper to a SilicaMicrosphere Whispering-Gallery Mode System, Phys Rev Lett, 85 (2000) 74-77.

[35] S. Huang, Z. Zhou, D. Li, T. Liu, X. Wang, J. Zhu, Y. Li, Compact broadband acoustic sink with coherently coupled weak resonances, Sci Bull, (2019).

[36] J. Li, W. Wang, Y. Xie, B.-I. Popa, S.A. Cummer, A sound absorbing metasurface with coupled resonators, Appl Phys Lett, 109 (2016) 091908. 